\title{
Piperine ameliorates the severity of fibrosis via inhibition of TGF-/S/SMAD signaling in a mouse model of chronic pancreatitis
}

\author{
JI-WON CHOI ${ }^{1,2^{*}}$, SUNG-KON LEE ${ }^{1 *}$, MYOUNG-JIN KIM ${ }^{1}$, DONG-GU KIM ${ }^{2}$, JOON-YEON SHIN ${ }^{1}$, \\ ZIQI ZHOU ${ }^{1}$, IL-JOO JO ${ }^{3}$, HO-JOON SONG ${ }^{1}$, GI-SANG BAE ${ }^{2,4}$ and SUNG-JOO PARK ${ }^{1,2}$ \\ ${ }^{1}$ Department of Herbology, School of Korean Medicine; ${ }^{2}$ Hanbang Cardio-Renal Syndrome Research Center; \\ ${ }^{3}$ Division of Beauty Sciences, School of Natural Sciences; ${ }^{4}$ Department of Pharmacology, \\ School of Korean Medicine, Wonkwang University, Iksan, Jeonbuk 54538, Republic of Korea
}

Received April 12, 2019; Accepted August 12, 2019

DOI: $10.3892 / \mathrm{mmr} .2019 .10635$

\begin{abstract}
Chronic pancreatitis (CP) is characterized by recurrent pancreatic injury, resulting in inflammation and fibrosis. Currently, there are no drugs for the treatment of pancreatic fibrosis associated with CP. Piperine, a natural alkaloid found in black pepper, has been reported to show anti-inflammatory, anti-oxidative, and antitumor activities. Although piperine exhibits numerous properties in regards to the regulation of diverse diseases, the effects of piperine on $\mathrm{CP}$ have not been established. To investigate the effects of piperine on $\mathrm{CP}$ in vivo, we induced $\mathrm{CP}$ in mice through the repetitive administration of cerulein $(50 \mu \mathrm{g} / \mathrm{kg})$ six times at 1 -h intervals, 5 times per week, for a total of 3 weeks. In the pre-treatment groups, piperine
\end{abstract}

Correspondence to: Professor Sung-Joo Park, Department of Herbology, School of Korean Medicine, Wonkwang University, 460 Iksandaero, Iksan, Jeonbuk 54538, Republic of Korea

E-mail:parksj08@wku.ac.kr

Professor Gi-Sang Bae, Department of Pharmacology, School of Korean Medicine, Wonkwang University, 460 Iksandaero, Iksan, Jeonbuk 54538, Republic of Korea

E-mail: baegs888@wku.ac.kr

*Contributed equally

Abbreviations: $\alpha$-SMA, $\alpha$-smooth muscle actin; BSA, bovine serum albumin; CCL, C-C motif chemokine ligand; CXCL, $\mathrm{C}-\mathrm{X}-\mathrm{C}$ motif chemokine ligand; $\mathrm{CP}$, chronic pancreatitis; $\mathrm{ECM}$, extracellular matrix; DAPI, 4',6-diamidino-2-phenylindole; HPRT, hypoxanthine-guanine phosphoribosyltransferase; H\&E, hematoxylin and eosin; HRP, horseradish peroxidase; ICD, International Classification of Disease; IHC, immunohistochemistry; IL, interleukin; PBST, phosphate-buffered saline/Tween 20; PSCs, pancreatic stellate cells; RAP, recurrent acute pancreatitis; RT, room temperature; RT-qPCR, reverse transcription-quantitative polymerase chain reaction; SDS, sodium dodecyl sulfate; TGF- $\beta$, transforming growth factor- $\beta$; TNF, tumor necrosis factor

Key words: chronic pancreatitis, piperine, inflammation, fibrosis, transforming growth factor- $\beta$
$(1,5$, or $10 \mathrm{mg} / \mathrm{kg})$ or corn oil were administrated orally at $1 \mathrm{~h}$ before the first cerulein injection, once a day, 5 times a week, for a total of 3 weeks. In the post-treatment groups, piperine $(10 \mathrm{mg} / \mathrm{kg})$ or corn oil was administered orally at 1 or 2 week after the first cerulein injection. Pancreases were collected for histological analysis. In addition, pancreatic stellate cells (PSCs) were isolated to examine the anti-fibrogenic effects and regulatory mechanisms of piperine. Piperine treatment significantly inhibited histological damage in the pancreas, increased the pancreatic acinar cell survival, reduced collagen deposition and reduced pro-inflammatory cytokines and chemokines. In addition, piperine treatment reduced the expression of fibrotic mediators, such as $\alpha$-smooth muscle actin ( $\alpha$-SMA), collagen, and fibronectin 1 in the pancreas and PSCs. Moreover, piperine treatment reduced the production of transforming growth factor (TGF)- $\beta$ in the pancreas and PSCs. Furthermore, piperine treatment inhibited TGF- $\beta$-induced pSMAD2/3 activation but not pSMAD1/5 in the PSCs. These findings suggest that piperine treatment ameliorates pancreatic fibrosis by inhibiting TGF- $\beta /$ SMAD $2 / 3$ signaling during $\mathrm{CP}$.

\section{Introduction}

Chronic pancreatitis (CP) (ICD-10 code for recurrent chronic pancreatitis: K86.1) is a progressive, irreversible disease process characterized by chronic inflammation, glandular necrosis and fibrosis, which leads to the impairment of pancreatic exocrine and endocrine functions (1). Patients with CP suffer from chronic abdominal pain, impaired digestion, malnutrition, anorexia, diabetes and disease-related complications, such as the formation of pseudocysts, which negatively impact their quality of life (2). In the USA, the prevalence of CP has been estimated to be $20-30$ cases per $10^{5}$ individuals in the entire population $(3,4)$. Although general and statistical reports concerning the prevalence of $\mathrm{CP}$ do not exist in the Republic of Korea, we predict that the prevalence of $\mathrm{CP}$ in Korea may be $35-40$ cases per $10^{5}$ individuals from the reports of Japanese individuals (5). The mortality rate of CP patients is higher than that of the general population, and the survival at 10 years after the onset of CP is estimated at $69-80 \%(6,7)$. The leading cause of death includes pancreatic cancer, with a higher incidence observed in CP patients (8). The development 
of pancreatic cancer may stem from the pancreatic inflammatory process, and it is known that they have common risk factors such as alcohol and smoking.

In the present study, a mouse model of repetitive acute pancreatitis (RAP)-induced CP was used. This model is widely used because of its ease of induction and good reproducibility. The RAP mouse model is characterized histologically by extensive inflammatory infiltrate, acinar cell atrophy and pancreatic fibrotic lesions. Moreover, it exhibits the biochemical and pathophysiological features of CP observed in humans, such as fibrotic destruction of the pancreatic gland, and structural impairment of exocrine and endocrine functions $(9,10)$. Accumulating genetic, clinical and experimental evidence supports the hypothesis that $\mathrm{CP}$ is the result of multiple episodes of RAP (11). Although many studies have been conducted to elucidate the pathophysiology of $\mathrm{CP}$, the pathobiochemical and molecular mechanisms remain unclear. Currently, no significant prophylactic or therapeutic agents are clinically effective for the prevention of CP.

Pancreatic stellate cells (PSCs) play a crucial role in the initiation and progression of pancreatic fibrogenesis in CP (12). Under normal conditions, these cells exist in the quiescent state. Once a cell is injured, these quiescent cells convert to myofibroblast-like, $\alpha$-smooth actin ( $\alpha$-SMA)-positive cells that are highly proliferative and capable of depositing fibrillar collagen in the interstitial spaces (13). The representative stimulatory cytokine found on PSCs is transforming growth factor (TGF)- $\beta$, which is generated by inflammatory mediators (14). TGF- $\beta$ stimulates PSCs to synthesize and secrete matrix proteins such as collagen and fibronectin (9). Thus, the regulation of TGF- $\beta$ in the development of $\mathrm{CP}$ is a key factor for the treatment of $\mathrm{CP}$.

Piperine is a phenolic component of black pepper (Piper nigrum) and long pepper (P. longum) (15), and has many beneficial biological and pharmacological effects, such as anti-depressant, hepatoprotective, anti-metastatic and anti-thyroid effects (16). We previously reported the protective effects of piperine against acute pancreatitis and sepsis $(17,18)$. However, currently, it is unclear whether piperine can attenuate pancreatic fibrosis during CP. Therefore, we aimed to determine the anti-inflammatory and anti-fibrotic effects of piperine on cerulein-induced CP in mice. We examined histological variations in the pancreas, and typical characteristics such as activation of PSCs, deposition of extracellular matrix (ECM), and production of cytokines (TNF- $\alpha$, IL-1 $\beta$, IL-6 and TGF- $\beta$ ) and chemokines (CCL2 and CXCL2). Furthermore, using primary mouse PSCs, the effects of piperine on TGF- $\beta$-induced PSC activation and ECM formation were demonstrated.

\section{Materials and methods}

Materials. Piperine, corn oil, cerulein, DAPI, hematoxylin, eosin, xylene and ethanol were purchased from Sigma-Aldrich/Merck KGaA. Easy-Blue ${ }^{\text {TM }}$ Total RNA extraction kit and RIPA buffer were purchased from iNtRON Biotechnology (Seongnam, Korea). Antibodies against phospho-specific SMAD2/3 (cat. no. sc-11769), $\alpha$-SMA (cat. no. sc-32251), amylase (cat. no. sc-46657) and GAPDH (cat. no. sc-32233) were purchased from
Santa Cruz Biotechnology (Santa Cruz, CA, USA). TGF- $\beta$ (cat. no. ab66043) and collagen I (cat. no. ab34710) were purchased from Abcam (Cambridge, MA, UK). Phospho-specific SMAD1/5 (cat. no. 9516S), SMAD3 (cat. no. 9523S), and SMAD5 (cat. no. 9517S) were purchased from Cell Signaling Technology (Beverly, MA, USA).

Animals. All experiments were performed according to protocols approved by the Animal Care Committee of Wonkwang University (WKU15-18). In total, 180 female C57BL/6 mice (6-8 weeks of age, weighing 15-20 g) were purchased from Orient Bio (Sungnam, KyungKiDo, Korea). All animals were bred and housed in standard shoebox cages in a climate-controlled room with an ambient temperature of $23 \pm 2^{\circ} \mathrm{C}$ and a 12 -h light-dark cycle for 7 days. Animals were fed standard laboratory chow, allowed water ad libitum, and randomly assigned to a control or experimental group.

Experimental design. CP was induced by administering intraperitoneal injections of cerulein $(50 \mu \mathrm{g} / \mathrm{kg}, \mathrm{n}=6$ per group for three experiments, total=18) six times at 1 -h intervals, 5 times per week, for a total of 3 weeks. In the pre-treatment groups, piperine $(1,5$, or $10 \mathrm{mg} / \mathrm{kg}, \mathrm{n}=6$ per group for three experiments, total=18) or corn oil (control group, $n=6$ per group for three experiments, total=18) was administrated orally $1 \mathrm{~h}$ before the first cerulein injection, once a day, 5 times a week, for a total of 3 weeks. In the post-treatment groups, piperine $(10 \mathrm{mg} / \mathrm{kg}, \mathrm{n}=6$ per group for three experiments, total=18) or corn oil was administered orally at 1 or 2 weeks after the first cerulein injection. Mice were sacrificed at $24 \mathrm{~h}$ after the last cerulein injection via $\mathrm{CO}_{2}$ asphyxiation. Pancreases were collected for further analysis. All experiments were performed independently for three times.

Histology. The pancreatic tissues were fixed overnight in $4 \%$ paraformaldehyde, embedded in paraffin using standard methods, and cut into serial sections (4- $\mu \mathrm{m}$ thick). The tissues were stained with hematoxylin and eosin (H\&E) for histological examination via light microscopy. The tissue slides were blind-labeled and reviewed by a pancreas pathologist who had no knowledge of the experimental groups. The histopathological assessment of CP was scored based on inflammation and glandular atrophy. Using a previously described method (19), a tissue section representing a minimum of 100 fields was examined for each sample and scored on a scale of 0-3 (0 being normal and 3 being severe).

Immunofluorescence. Immunofluorescence assays for $\alpha$-SMA and TGF- $\beta$ was performed on frozen pancreatic tissue sections by using a cryostat microtome. The tissues were permeabilized with $0.1 \%$ Triton $\mathrm{X}-100$ for $15 \mathrm{~min}$ at room temperature (RT), and blocked with $3 \%$ BSA for $1 \mathrm{~h}$ at RT. The sections were then incubated with the primary antibodies against $\alpha$-SMA (1:500 dilution in 3\% BSA; Santa Cruz Biotechnology) and TGF- $\beta$ (1:500 dilution in 3\% BSA; Cell Signaling Technology) at $4^{\circ} \mathrm{C}$ overnight followed by the fluorescence-labeled secondary antibodies Alexa Fluor 594 goat anti-mouse (cat. no. A11005) and Alexa Fluor 568 goat anti-rabbit (cat. no. A11011) at RT for $1 \mathrm{~h}(1: 2,000$ dilution in 3\% BSA; Invitrogen; Thermo Fisher Scientific, Inc.). Nuclei were counterstained with DAPI 
(1:2,000 dilution in 3\% BSA; Vector Laboratories, Burlingame, CA, USA) for $5 \mathrm{~min}$ at RT. Stained sections were visualized using a confocal laser microscope (Olympus, Japan).

Collagen staining. Sirius Red was used for staining of the collagen deposition. Deparaffinized and rehydrated slides were immersed in saturated picric acid for 20 min and rinsed in tap water until the yellow stain in the specimens was cleared. The slides were then stained with $0.1 \%$ Sirius Red solution $(0.5 \mathrm{~g}$ of Direct Red 80; Sigma-Aldrich; Merck KGaA) in $500 \mathrm{ml}$ of picric acid for $1 \mathrm{~h}$, washed in two changes of acidified water ( $5 \mathrm{ml}$ glacial acetic acid in 11 of water), and counterstained with hematoxylin. The relative intensity was measured using Leica microscopy software (Wetzlar, Germany).

Reverse transcription-quantitative polymerase chain reaction $(R T-q P C R)$. Total RNA was extracted using an Easy-Blue ${ }^{\mathrm{TM}}$ Total RNA extraction kit according to the manufacturer's instructions and reverse transcription of RNA to cDNA was performed using an ABI cDNA synthesis kit (Applied Biosystems, Foster City, CA, USA) (conditions: $37^{\circ} \mathrm{C}$ for $1 \mathrm{~h}$, followed by $95^{\circ} \mathrm{C}$ for $5 \mathrm{~min}$ ). TaqMan quantitative RT-PCR with an ABI StepOne Plus detection system was performed according to the manufacturer's instructions (Applied Biosystems; Thermo Fisher Scientific, Inc.). For each sample, triplicate test reactions and a control reaction without reverse transcriptase were analyzed for expression of the gene of interest and to control for variations in the reactions. All qPCR data were normalized to levels against the housekeeping gene hypoxanthine guanine phosphoribosyltransferase (HPRT). Forward, reverse, and probe oligonucleotide primers for multiplex real-time TaqMan PCR were purchased from ABI (Applied Biosystems; Thermo Fisher Scientific, Inc.). Cycling conditions were $50^{\circ} \mathrm{C}$ for $2 \mathrm{~min}, 95^{\circ} \mathrm{C}$ for $10 \mathrm{~min}$, followed by 40 cycles of $95^{\circ} \mathrm{C}$ for $10 \mathrm{sec}$ and $60^{\circ} \mathrm{C}$ for $30 \mathrm{sec}$. The data were analyzed using StepOne ${ }^{\mathrm{TM}}$ software (version 2.3; Applied Biosystems; Thermo Fisher Scientific, Inc.). The $2^{-\Delta \Delta \mathrm{Cq}}$ method was used to determine the relative mRNA expression level (20).

Isolation of mouse PSCs. PSCs were isolated from the C57BL/6 mice via digestion of the pancreatic tissue and Nycodenz ${ }^{\circledR}$ (Sigma-Aldrich; Merck KGaA) density gradient centrifugation, as described previously (11). Briefly, freshly isolated mouse PSCs were cultured in DMEM/High Glucose supplemented with $10 \%$ fetal bovine serum and $1 \%$ penicillin-streptomycin at $37^{\circ} \mathrm{C}$ with $5 \% \mathrm{CO}_{2}$. Passages 3 to 6 were used.

Western blot analysis. PSCs were harvested and then lysed with lysis buffer (1\% cocktail of protease inhibitor and $1 \%$ phosphatase inhibitor in 1X RIPA buffer). The cells were boiled in sample buffer [62.5 mM Tris-HCl, pH 6.8, 2\% sodium dodecyl sulfate (SDS), $20 \%$ glycerol, and 10\% 2-mercaptoethanol]. The protein concentrations were determined by bicinchoninic acid assay. Total cell proteins $(20 \mu \mathrm{g})$ were separated via $10 \%$ SDS-polyacrylamide gel and transferred to a PVDF membrane (GE Healthcare Life Sciences, Little Chalfont, Buckinghamshire, UK). The membranes were blocked with 5\% skim milk in PBS-Tween-20 (PBST) for $2 \mathrm{~h}$ at RT and incubated with primary antibodies $(1: 1,000)$ at $4^{\circ} \mathrm{C}$ overnight: $\alpha$-SMA, collagen I, GAPDH, pSMAD2/3, pSMAD1/5,
SMAD3, SMAD5. After washing thrice, the membranes were incubated with HRP-conjugated goat anti-mouse (1:5,000; cat. no. 1031-05; Southern Biotech, Birmingham, AL, USA) or goat anti-rabbit (1:5,000; cat. no. NB7156; Novus Biologicals, LLC, Littleton, CO, USA) IgG secondary antibodies for $1 \mathrm{~h}$ at room temperature. The protein bands were visualized using an enhanced chemiluminescence detection system (Amersham; GE Healthcare), according to the manufacturer's recommended protocol. Capture of protein bands and quantitative analysis were performed using Quantity One ${ }^{\circledR}$ software version 4,6,6 (Bio-Rad Laboratories, Inc, Hercules, CA, USA).

Immunohistochemical analysis. Immunohistochemical staining for amylase was performed using DAB immunohistochemical kit (Dako, Cytomation, Denmark; as shown in Fig. S1).

Statistical analysis. Results are expressed as the mean \pm standard error of the mean (SEM). Significance was evaluated using a two-way analysis of variance (ANOVA), with time and dose parameters. Significant ANOVA tests were further examined by post hoc analysis, using the Duncan method as a multiple comparison among groups. Values of $\mathrm{P}<0.05$ were accepted as statistically significant.

\section{Results}

Morphological and histological examination of the effects of piperine on the pancreases with $C P$. To determine whether piperine can attenuate the development of $\mathrm{CP}$, the histological architecture of the pancreas was investigated. Piperine had no adverse or harmful effects on animals and no significant alterations were found between the corn oil group and the piperine group with respect to the histology of the pancreatic tissues and body weights (data not shown). In the cerulein-induced CP mice, the histological features of the pancreases showed severe pancreatic damage such as glandular atrophy and inflammatory cell infiltration (Fig. 1B and C). However, these features were inhibited by treatment with piperine in a dose-dependent manner (Fig. 1B and C). To further examine the effect of piperine on $\mathrm{CP}$, the survival of pancreatic acini, which secrete amylase, were assessed. Mice with CP showed fewer pancreatic acini as indicated by the reduced levels of amylase. However, piperine treatment significantly reduced acinar cell death and destruction in the pancreases of the CP-induced mice (Fig. S1).

Effect of piperine on the activation of PSCs during CP. Generally, PSCs are desmin-positive cells. When PSCs are activated they transform into myofibrolast-like cells expressing $\alpha$-SMA (21). Therefore, we examined the expression of $\alpha$-SMA as a marker of activated PSCs in the pancreas using immunofluorescence analysis. The immunofluorescence analysis revealed that the number of $\alpha$-SMA-positive cells (in red color) were markedly increased in the CP-induced mice. Piperine-treated mice showed decreased $\alpha$-SMA expression (Fig. 2A and B). To confirm the above finding, the mRNA expression of Acta- 2 for $\alpha$-SMA was assessed. Acta- 2 level was markedly increased in the pancreas of the CP-induced mice. However, this increase was significantly reduced by treatment with piperine (Fig. 2C). 
A

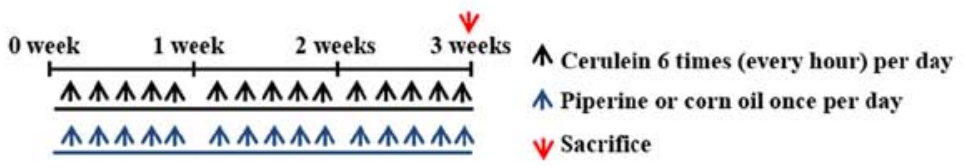

B CP
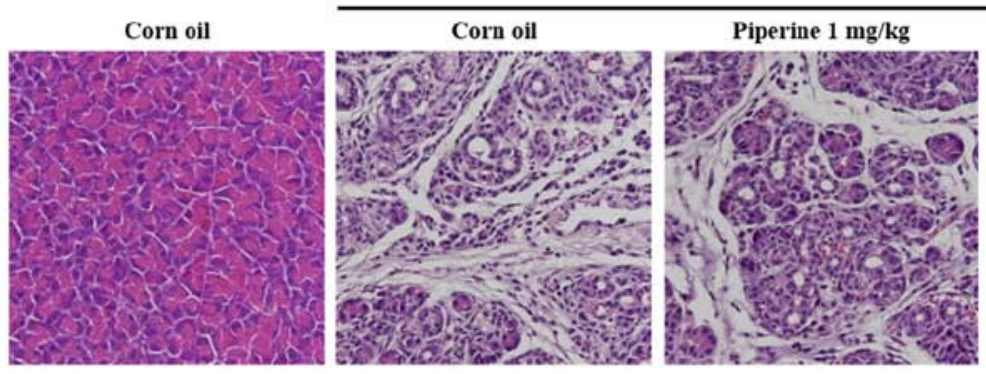

Piperine $5 \mathrm{mg} / \mathrm{kg}$

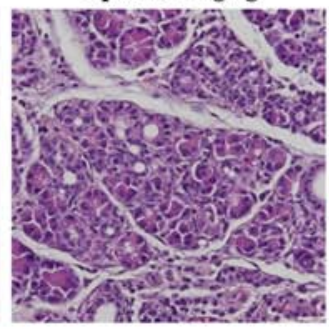

Piperine $10 \mathrm{mg} / \mathrm{kg}$

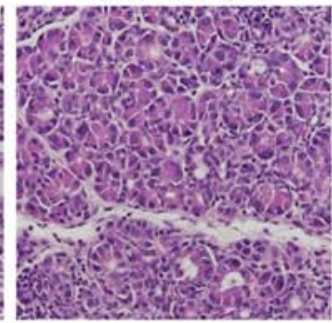

C
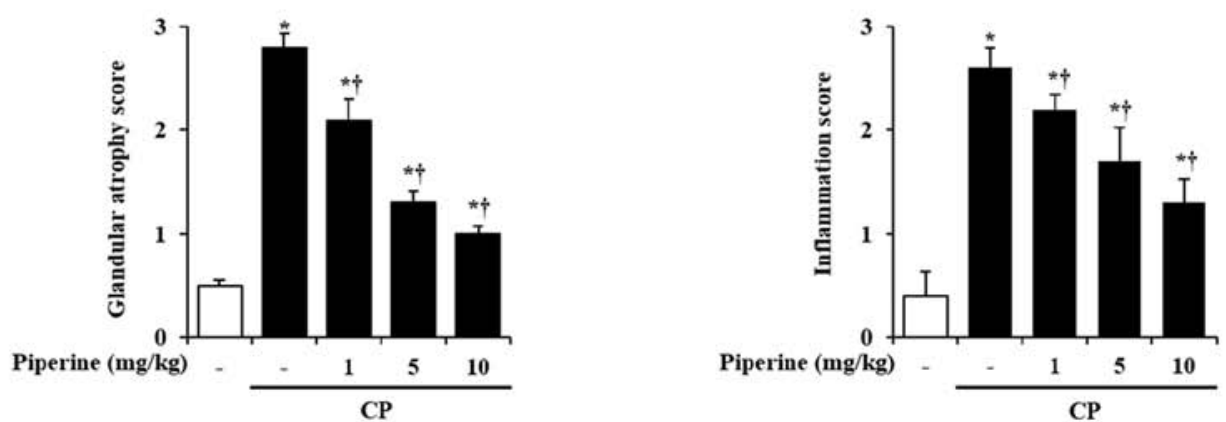

Figure 1. Effects of piperine on pancreatic damage caused by cerulein-induced $\mathrm{CP}$ in mice. (A) $\mathrm{CP}$ was induced by the administration of six intraperitoneal injections of cerulein $(50 \mu \mathrm{g} / \mathrm{kg})$ at 1-h intervals, 5 days per week, for a total of 3 weeks. Piperine or corn oil was administrated orally at $1 \mathrm{~h}$ before the first cerulein injection, once a day, 5 times a week for a total of 3 weeks. The groups of mice were euthanized 3 weeks after the beginning of the cerulein treatment. (B) Representative H\&E-stained pancreatic tissue sections of the control mice and piperine-treated mice $(1,5 \mathrm{or} 10 \mathrm{mg} / \mathrm{kg})$ before the first cerulein $(50 \mu \mathrm{g} / \mathrm{kg}$ )-mediated induction of CP. (C) Histological sections of the pancreas were scored from 0 (normal) to 3 (severe) for inflammation and glandular atrophy. Data are represented as means \pm SEM for 6 mice in each group. Results are representative of three experiments. ${ }^{*} \mathrm{P}<0.05$ vs. corn oil alone; ${ }^{\dagger} \mathrm{P}<0.05$ vs. CP. Original magnification, x200. CP, chronic pancreatitis; H\&E, hematoxylin and eosin.

Effect of piperine on ECM production during CP. ECM deposition such as collagen and fibronectin is an important manifestation of CP. To determine the degree of collagen deposition following piperine treatment, Sirius Red staining was performed. As shown in Fig. 3A, CP-induced mice showed a marked increase in collagen deposition in the pancreas. However, a significant decrease in collagen deposition was observed in the piperine-treated mice (Fig. 3A and B). Furthermore, the mRNA levels of collagen I and fibronectin 1 were also reduced following treatment with piperine (Fig. 3C and D).

Effect of piperine on cytokine and chemokine production during $C P$. It has been reported that TGF- $\beta$ contributes to chronic pancreatic injury and fibrosis (22). Therefore, the expression of TGF- $\beta$ was measured in pancreases of the CP-induced mice. Pancreatic TGF- $\beta$ was markedly increased by $\mathrm{CP}$ as previously reported (22); however, piperine treatment significantly inhibited the TGF- $\beta$ expression (Fig. 4A and B). The level of TGF- $\beta$ mRNA was also inhibited by piperine while corn oil-treated mice with $\mathrm{CP}$ showed higher levels of TGF- $\beta$ mRNA (Fig. 4C). In addition, cytokines such as TNF- $\alpha$, IL- $1 \beta$, and IL- 6 and chemokines such as CCL2 and CXCL2 are important candidate factors that may activate PSCs to promote CP $(22,23)$. Thus, we investigated whether administration of piperine is able to decrease the cytokine and chemokine levels during CP. The results of this experiment (Fig. 4D and E) demonstrated that the treatment of piperine decreased the levels of cytokines such as TNF- $\alpha$, IL-1 $\beta$, and IL-6 mRNA and chemokines such as CCL2 and CXCL2 mRNA (Fig. 4D and E).

Effect of piperine on TGF- $\beta$-induced PSC activation in the isolated PSCs. To evaluate the detailed beneficial effects of piperine against $\mathrm{CP}$, mouse PSCs, the major cells which regulate $\mathrm{CP}$, were isolated. As previous studies have shown that TGF- $\beta$ promotes PSC activation, and stimulates ECM synthesis $(24,25)$, we examined the effect of piperine on the TGF- $\beta$-induced expression of fibrosis-related genes such as $\alpha$-SMA, fibronectin 1 and collagen I/III in isolated PSCs. Using real-time PCR analysis, we found that, compared with the control group, mRNA levels of $\alpha$-SMA, fibronectin 1 and collagen I/III in the TGF- $\beta$ treatment group were notably increased. However, the increased mRNA expression by TGF- $\beta$ was significantly decreased in the presence of piperine (Fig. 5A-D). Likewise, protein levels of $\alpha$-SMA and collagen I were also decreased by piperine treatment (Fig. 5E).

TGF- $\beta$ intracellular signaling is mediated and modulated primarily by SMADs $(26,27)$. Thus, to ascertain the 
A

CP
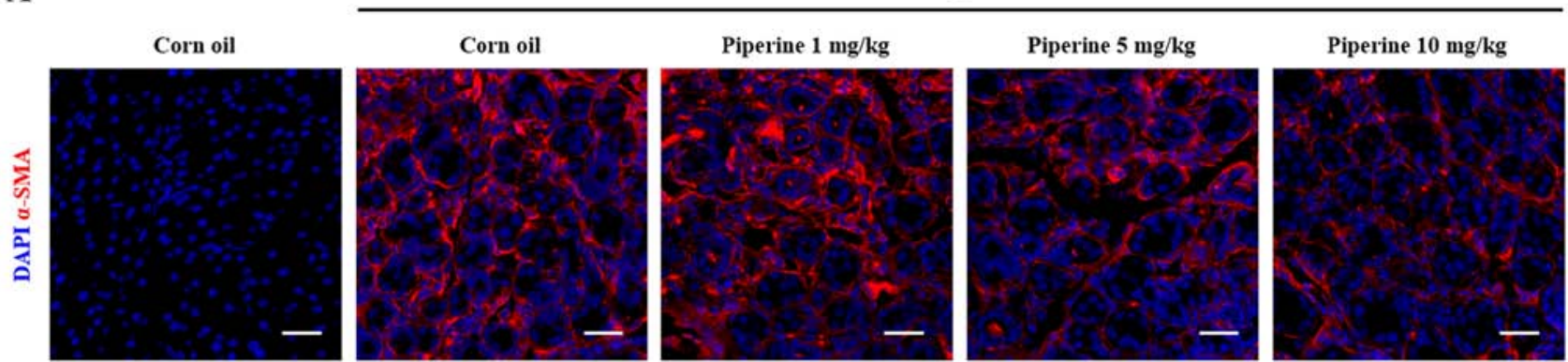

B

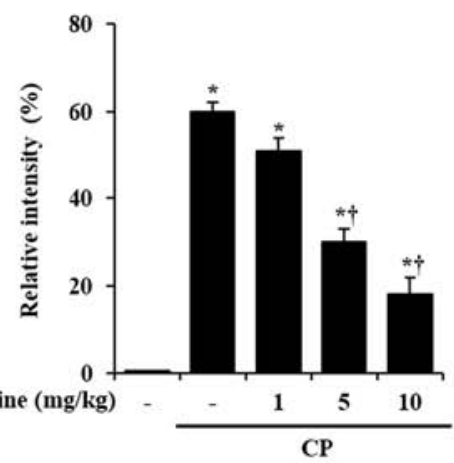

C

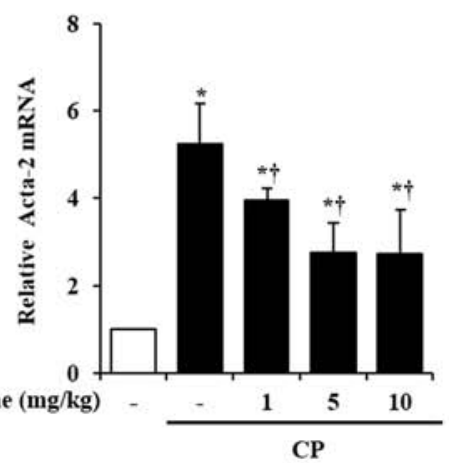

Figure 2. Effect of piperine on PSC activation during CP. (A) Confocal images of immunofluorescence staining of $\alpha$-SMA (red) and DAPI (blue). (B) Relative intensity of $\alpha$-SMA. (C) Acta- 2 mRNA was assessed using RT-qPCR. Data are represented as means \pm SEM for 6 mice in each group. Results are representative of three experiments. "P $<0.05$ vs. corn oil alone; ${ }^{\dagger} \mathrm{P}<0.05$ vs. CP. Scale bar, $20 \mu \mathrm{m}$. PSC, pancreatic stellate cell; CP, chronic pancreatitis; $\alpha$-SMA, $\alpha$-smooth muscle actin; DAPI, 4',6-diamidino-2-phenylindole.

A

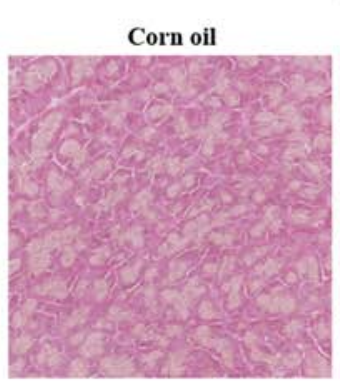

B

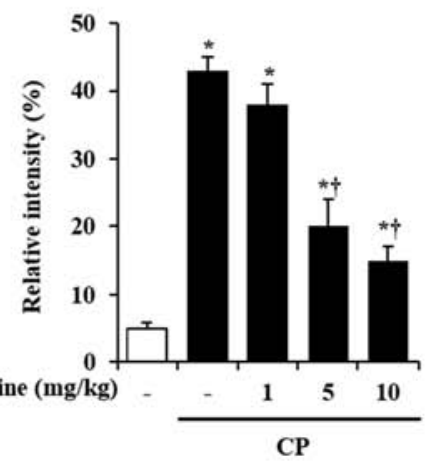

C

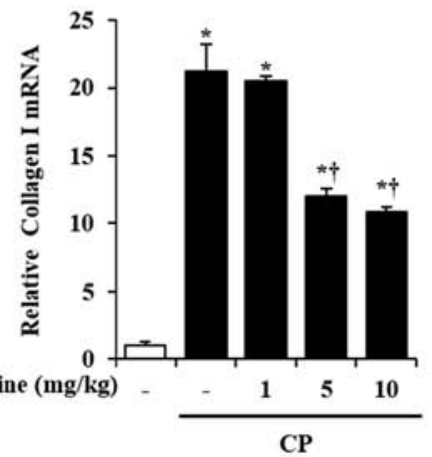

CP

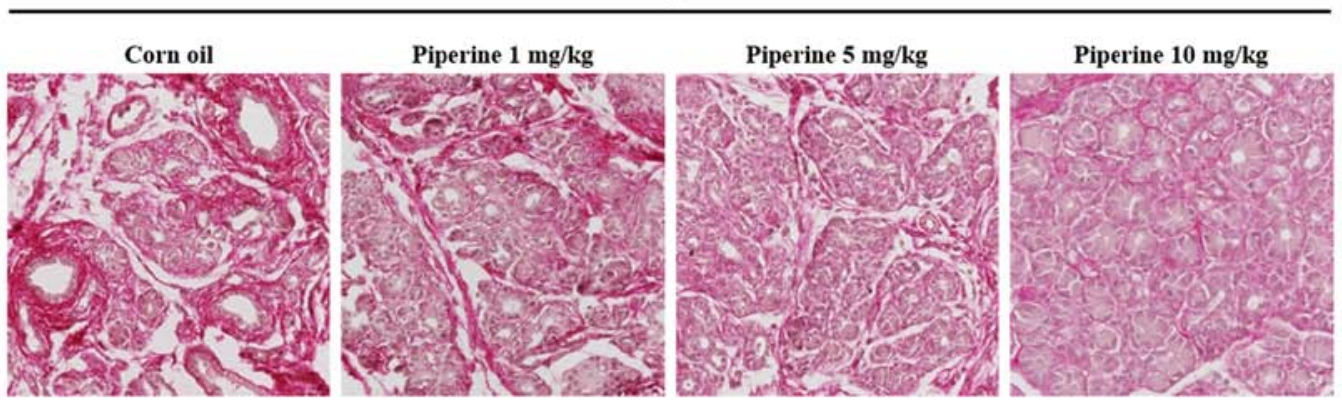

D

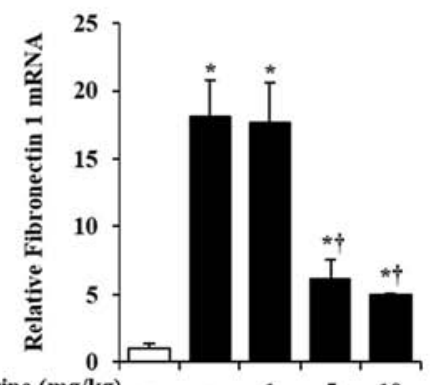

Piperine (mg/kg)

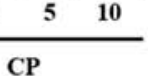

Figure 3. Effect of piperine on ECM deposition during CP. (A) Sirius red staining for collagen deposition. (B) Relative intensity of Sirius Red. The pancreatic mRNA levels of (C) collagen I and (D) fibronectin 1 were assessed using RT-qPCR. Data are represented as means \pm SEM for 6 mice in each group. Results are representative of three experiments. ${ }^{*} \mathrm{P}<0.05$ vs. corn oil alone; ${ }^{\dagger} \mathrm{P}<0.05$ vs. CP. Original magnification, $\mathrm{x} 200$. CP, chronic pancreatitis; ECM, extracellular matrix.

mechanisms responsible for the inhibition of PSC activation by piperine, we firstly examined the expression of TGF- $\beta$ and SMAD. In accordance with previous reports, TGF- $\beta$ and
SMAD2/3 and SMAD1/5 were increased in the activated PSCs. However, in the presence of piperine, TGF- $\beta$ expression and phosphorylation of SMAD2/3 were significantly 
A

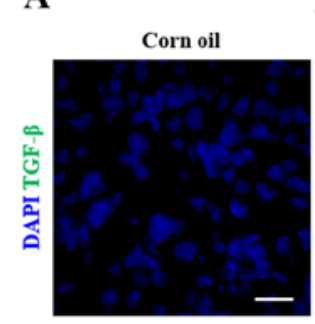

CP

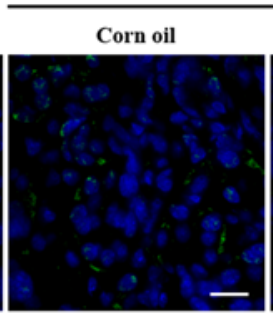

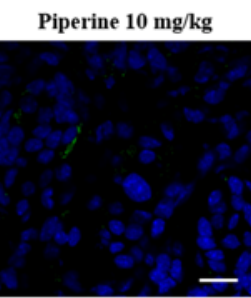

B

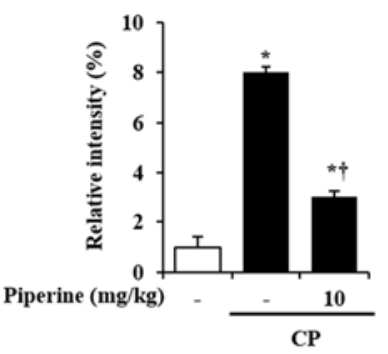

C

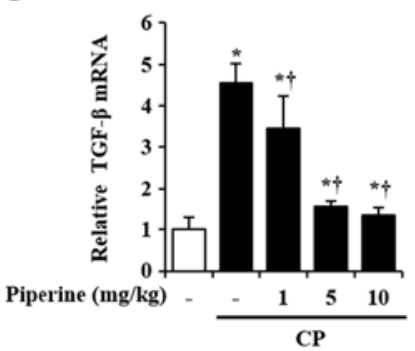

D
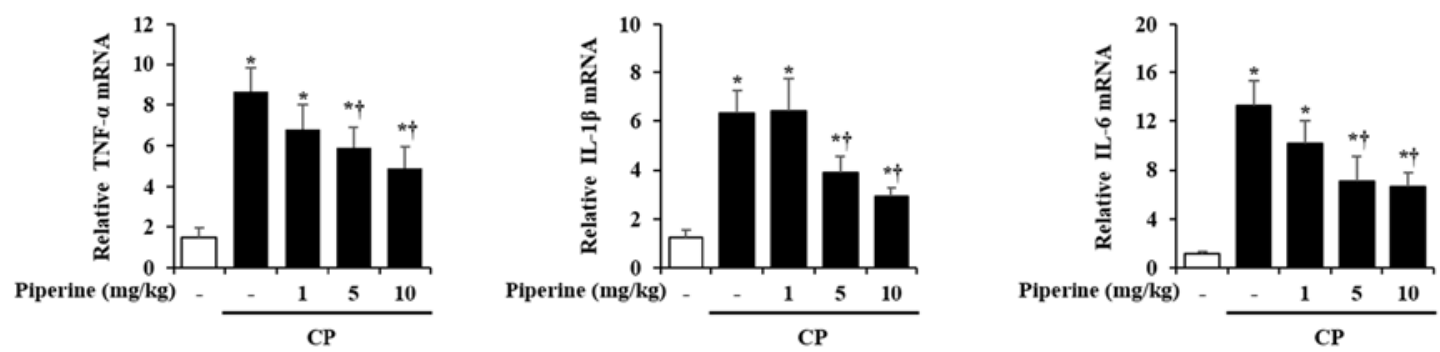

E
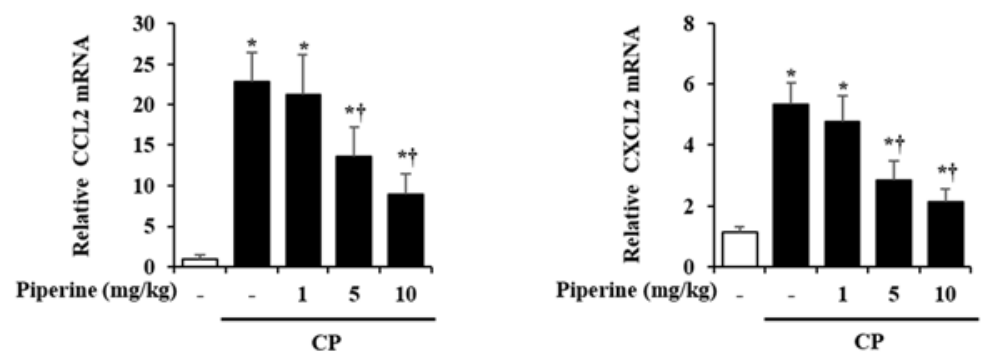

Figure 4. Effect of piperine on cytokines (TGF- $\beta$, TNF- $\alpha$, IL-1 $\beta$ and IL-6 mRNA) and chemokines (CCL2 and CXCL2 mRNA) production during CP. (A) Confocal images of immunofluorescence staining of TGF- $\beta$ (green) and DAPI (blue). (B) Relative intensity of TGF- $\beta$. (C) TGF- $\beta$ mRNA was determined using RT-qPCR. (C) TGF- $\beta$, (D) TNF- $\alpha$, IL-1 $\beta$ and IL-6, (E) CCL2 and CXCL2 mRNA was determined using RT-qPCR. Data are represented as means \pm SEM for 6 mice in each group. Results are representative of three experiments. ${ }^{*} \mathrm{P}<0.05$ vs. corn oil alone; ${ }^{\dagger} \mathrm{P}<0.05$ vs. CP. Scale bar, $10 \mu \mathrm{m}$. CCL, C-C motif chemokine ligand; CXCL, C-X-C motif chemokine ligand; CP, chronic pancreatitis; IL, interleukin; TGF- $\beta$, transforming growth factor- $\beta$; TNF, tumor necrosis factor; DAPI, 4',6-diamidino-2-phenylindole.

inhibited, while phosphorylation of SMAD1/5 was not altered (Fig. 5F and G).

Therapeutic effects of piperine during $C P$. To examine the therapeutic effects of piperine on $\mathrm{CP}$, we administered piperine orally at 1 week or 2 week after the onset of CP (Fig. 6A). Thus, piperine was administered for 2 weeks (starting at 1 week after the onset of $\mathrm{CP}$ ) or 1 week (starting at 2 week after the onset of $\mathrm{CP}$ ) during $\mathrm{CP}$. Then, the mice were sacrificed at 3 weeks after the beginning of the first cerulein injection. Injury of the pancreas such as glandular atrophy and inflammatory cell infiltration was significantly attenuated by post-treatment of piperine during $\mathrm{CP}$ (Fig. 6B and C). Furthermore, the elevation of $\alpha$-SMA, collagen deposition, fibronectin 1 and TGF- $\beta$ was inhibited by post-treatment of piperine during $\mathrm{CP}$ (Fig. $6 \mathrm{D}-\mathrm{K})$.

\section{Discussion}

Chronic pancreatitis $(\mathrm{CP})$ is an irreversible process that occurs through the activation of inflammatory cascades and pancreatic fibrosis. According to the recurrent acute pancreatitis (RAP) hypothesis, CP is the result of repeated episodes of AP. This cascade of events is initiated by acinar cell injury. Patient can recover from singular AP events. However, recurrent events amplify the degree of injury and inflammation, interrupt the repair process, and promote the progression of $\mathrm{CP}$. In our previous report (17), piperine, a natural alkaloid found in black pepper, reduced the severity of AP as determined by the reduction in pancreatic injury, acinar cell death, neutrophil infiltration, digestive enzyme levels and cytokine production. Therefore, we examined the effect of piperine on the severity of CP using an RAP mouse model. In the present study, it was shown that the administration of piperine reduced the severity of $\mathrm{CP}$ as well as inflammation and fibrosis by inhibiting TGF- $\beta /$ SMAD signaling.

Piperine is a well-known phenolic compound that has various biological functions. Various studies have shown that piperine plays a role in inflammatory diseases such as neuronal diseases (28), arthritis (29), cardiovascular diseases (30), gastrointestinal disorders (31), sepsis (18) and acute pancreatitis (17). In a recent report (32), a high-carbohydrate, high-fat diet supplemented with piperine reduced fibrosis in rat livers. In addition, another study investigated the antioxidant effect of curcumin with piperine in tropical pancreatitis (33). However, it remains unclear as to whether piperine exerts preventive effects on inflammation and fibrosis in the pancreas during CP. In our previous report (17), there were no significant differential effects between the oral and intraperitoneal 
A

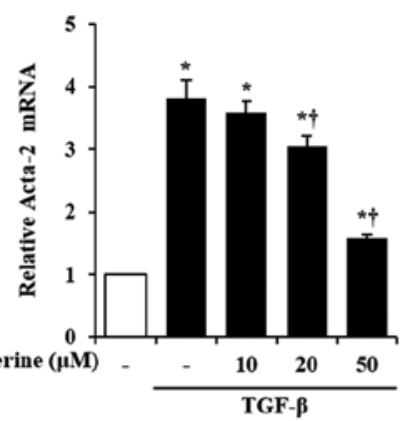

C

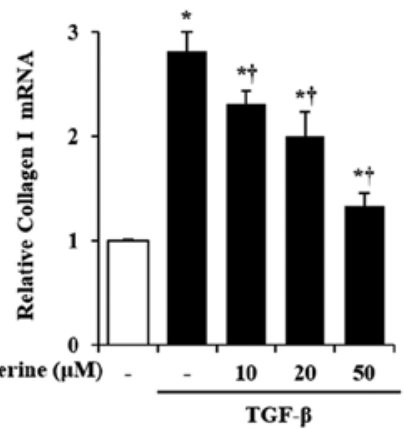

B

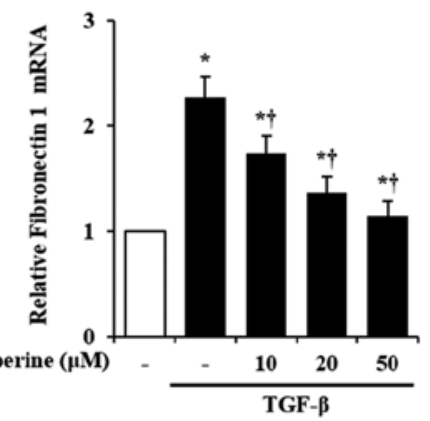

D

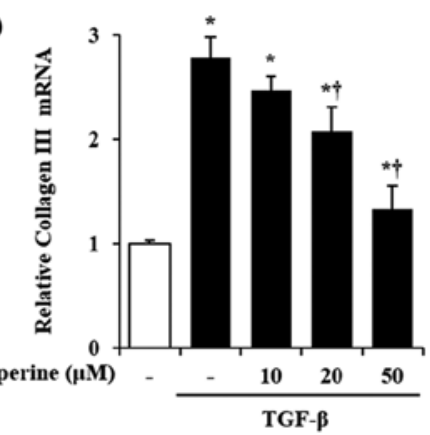

E
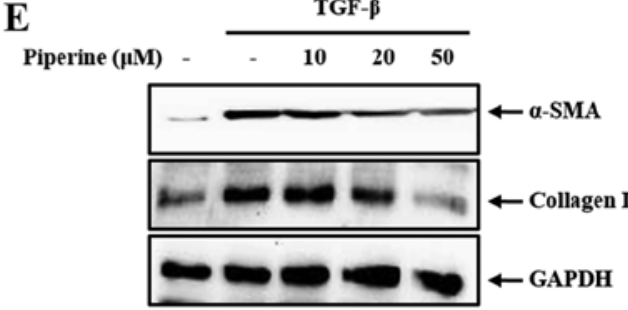

G

Piperine $(\mu \mathrm{M})$

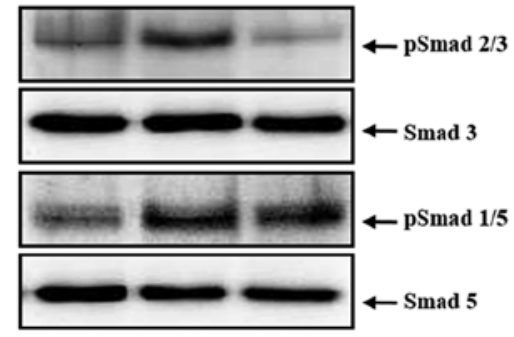

Figure 5. Effect of piperine on TGF- $\beta$-induced PSC activation and ECM production on the isolated PSCs. Mouse PSCs were pretreated with piperine at various concentrations $(10,20,50 \mu \mathrm{M})$ for $1 \mathrm{~h}$, and then stimulated with TGF- $\beta(0.5 \mathrm{ng} / \mathrm{ml})$ for $24 \mathrm{~h}$. Levels of mRNA were quantified by RT-PCR for (A) $\alpha$-SMA, (B) fibronectin 1, (C) collagen I, (D) collagen III, (F) TGF- $\beta$. (E) Protein levels of $\alpha$-SMA and collagen I were assessed using western blot analysis and GAPDH was used as a loading control. Mouse PSCs were pretreated with piperine $(50 \mu \mathrm{M})$ for $1 \mathrm{~h}$ followed by TGF- $\beta(0.5 \mathrm{ng} / \mathrm{ml})$ for $30 \mathrm{~min}$, and then whole cell lysates were harvested. (G) The phosphorylation (p) levels of SMAD2/3 and SMAD 1/5 were analyzed by western blot analysis and SMAD3 and SMAD5 were used as loading controls. Data are represented as means \pm SEM for 6 mice in each group. Results are representative of three experiments. "P<0.05 vs. saline alone; ${ }^{\dagger} \mathrm{P}<0.05$ vs. TGF- $\beta$. TGF- $\beta$, transforming growth factor- $\beta$; PSCs, pancreatic stellate cells; ECM, extracellular matrix; CP, chronic pancreatitis; $\alpha$-SMA, $\alpha$-smooth muscle actin.

administration of piperine on the severity of acute pancreatitis. Therefore, in the present study, we used intraperitoneal injections of piperine. The pancreatic histological appearance of the piperine-treated group was comparable with that of the saline-treated group (data not shown). The 3 weeks of repeated cerulein administrations were sufficient to induce $\mathrm{CP}$ in mice which was proven by the presence of inflammation, pancreatic atrophy and fibrosis (Fig. 1). The administration of piperine inhibited inflammatory cell infiltration and glandular atrophy, and increased the number of amylase-positive cells in the pancreases of CP-induced mice (Fig. S1), suggesting that the inhibition of inflammation and acinar cell death by piperine may improve exocrine function.

Repetitive administration of cerulein aggravates acinar cell injury, which ultimately results in pancreatic fibrosis (34). During pancreatic fibrosis, PSCs become activated by extra stimuli, and the activated PSCs ( $\alpha$-SMA-positive cells) promote pancreatic fibrosis by producing and secreting extracellular matrix (ECM) components such as collagen and fibronectin (35-37). Thus, regulating the activation of PSCs and ECM production from activated PSCs are crucial to treat CP. In our present study, we confirmed the activation of PSCs by $\alpha$-SMA and ECM deposition such as collagen and fibronectin. While corn oil-treated mice with CP showed a significant increase in $\alpha$-SMA-positive cells, piperine-treated mice with $\mathrm{CP}$ exhibited a lower number of $\alpha$-SMA positive cells and decreased ECM production (Figs. 2 and 3). These results suggest that piperine attenuates the severity of pancreatic fibrosis by inhibition of the generation and activation of PSCs, and deactivation of PSCs by piperine may lead to inhibition of exudates such as ECM from activated PSCs.

TGF- $\beta$ is a multipotent cytokine and plays an integral role in regulating immune, inflammatory response, cell growth and differentiation (38). An excess of TGF- $\beta$ in repeatedly injured 


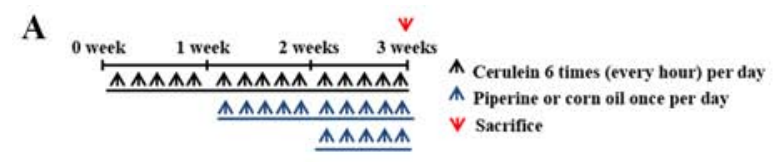

B
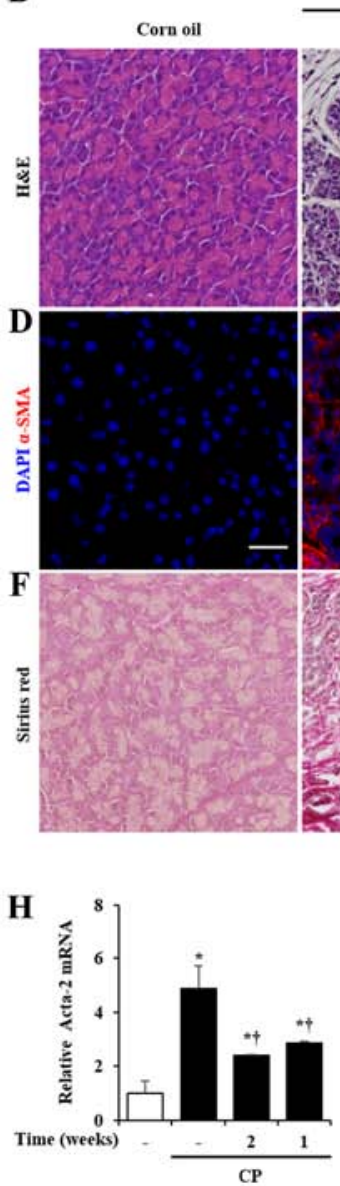

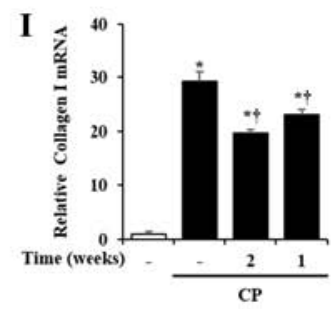

CP

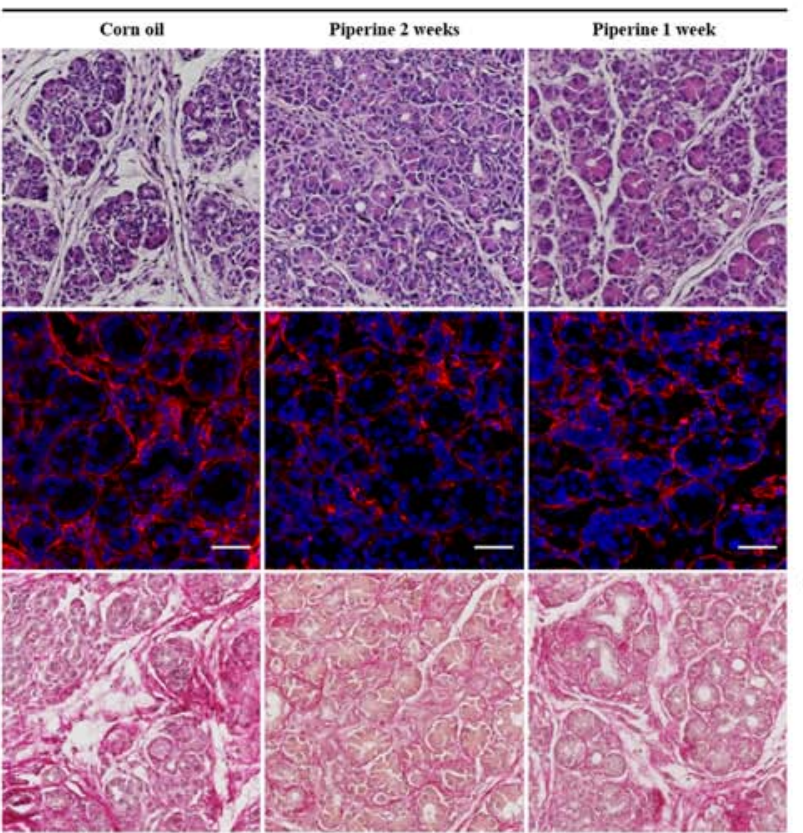

C

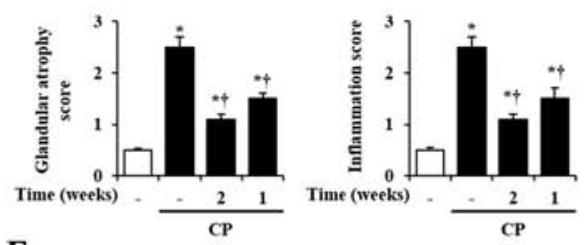

E
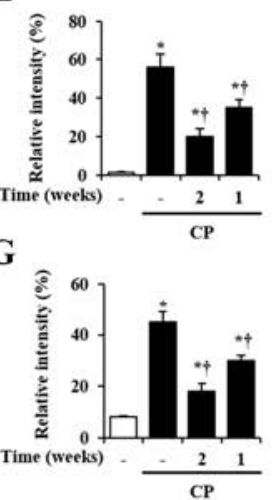

Figure 6. Therapeutic effects of piperine on cerulein-induced CP in mice. (A) CP was induced by the administration of six intraperitoneal injections of cerulein $(50 \mu \mathrm{g} / \mathrm{kg})$ at 1-h intervals, 5 days per week, for a total of 3 weeks. Piperine or corn oil was administrated orally 1 or 2 week after the onset of the first cerulein injection, once a day, 5 days per week, for a total of 2 or 1 week. Groups of mice were euthanized 3 weeks after the beginning of the cerulein treatment. (B) Representative H\&E-stained pancreatic tissue sections. (C) Histological sections of the pancreas were scored from 0 (normal) to 3 (severe) for glandular atrophy and inflammation. (D) Immunofluorescence staining of $\alpha$-SMA (red) and DAPI (blue). (E) Relative intensity of $\alpha$-SMA. (F) Sirius red staining. (G) Relative intensity of Sirius Red. The pancreatic mRNA levels of (H) Acta-2, (I) collagen I, (J) fibronectin 1 and (K) TGF- $\beta$ were determined using RT-qPCR. Data are represented as means \pm SEM for 6 mice in each group. Results are representative of three experiments. ${ }^{*} \mathrm{P}<0.05$ vs. corn oil alone; ${ }^{\dagger} \mathrm{P}<0.05$ vs. CP. Original magnification, x200. Scale bar, $20 \mu \mathrm{m}$. CP, chronic pancreatitis; H\&E, hematoxylin and eosin; $\alpha-S M A, \alpha-s m o o t h$ muscle actin; TGF- $\beta$, transforming growth factor- $\beta$; DAPI, 4',6-diamidino-2-phenylindol.

sites is thus associated with unresolved inflammation and fibrosis development. Previous reports have previously shown that excessive TGF- $\beta$ activity is the cause of increased scarring and fibrosis in diseases including hepatic cirrhosis (39) and cardiac fibrosis (40). TGF- $\beta$ may be involved in pancreatic fibrogenesis, which is released after cell injury and may be one of the pro-fibrogenic factors that promote fibrotic responses in PSCs $(34,36)$. PSCs are activated upon exposure to cytokines, such as TGF- $\beta$, and then $\alpha$-SMA is expressed, fibronectin 1 levels increase, and collagen is deposited near the fibrotic pancreatic tissues (41). Thus, in our experiments, we examined TGF- $\beta$ as a stimulator of PSCs during CP. In the present study, the mice with $\mathrm{CP}$ exhibited increased levels of TGF- $\beta$ in pancreatic tissue (Fig. 4A-C). When mice were treated with piperine during $\mathrm{CP}$, TGF- $\beta$ production was reduced in the mouse pancreas (Fig. 4A-C). These results suggest that piperine may negatively regulate PSC activation by inhibition of TGF- $\beta$ production. Based on these in vivo findings, we also directly examined the effect of piperine on TGF- $\beta$-induced PSC activation and ECM production from PSCs. It was demonstrated that piperine inhibited the expression of $\alpha$-SMA, fibronectin 1 and collagen I/III upon TGF- $\beta$ treatment, which suggests that piperine plays a key role in regulating fibrosis stimulated by TGF- $\beta$, as well as in vivo data (Fig. $5 \mathrm{~A}-\mathrm{E})$. We also aimed to examine the anti-fibrotic role of piperine on human PSCs to provide clinical value. Yet, it was not possible to obtain the human samples and permission for such a clinical study from our institution. Although a study using human PSCs was not carried out at this time, attainment of human samples will be achieved in future studies.

In the TGF- $\beta$-mediated signaling pathway, SMAD proteins are phosphorylated and activated by receptors and are 
translocated into the nucleus where these complexes regulate transcription of pro-fibrotic genes $(42,43)$. Among them, the TGF- $\beta /$ SMAD2/3 signaling pathway is reported to play a major role in the activation of PSCs. Studies have revealed that TGF- $\beta$ stimulates PSC activation in a SMAD2/3-dependent manner (44). A previous study demonstrated the ability to inhibit pancreatic fibrosis by blocking the TGF- $3 /$ SMAD2 signaling pathway (45). On the basis of the above-mentioned evidence, we investigated the effects of piperine on the expression of signaling in the TGF- $\beta /$ SMAD pathway. It was found that piperine treatment inhibited pSMAD2/3, but not pSMAD1/5 in PSCs (Fig. 5F and G). These data suggest that piperine reduces the expression of SMAD2/3 thereby downregulating the expression of PSC activation and ECM production. In addition, these results suggest that piperine exhibits its beneficial effects on PSCs by regulating the TGF- $\beta /$ SMAD pathway.

Taken together, this study demonstrated that piperine prevents the progression of pancreatic fibrosis by inhibiting the TGF- $\beta$ /SMAD signaling pathway. Our findings suggest that piperine has an anti-fibrotic effect against $\mathrm{CP}$ and may be useful for the clinical management of pancreatic disorders.

\section{Acknowledgements}

Not applicable.

\section{Funding}

This study was supported by the National Research Foundation of Korea (NRF) grant funded by the Korean government (MEST) (grant nos. NRF-2017R1C1B2010031, NRF-2017R1A5A2015805 and NRF-2019R1A2C2008814).

\section{Availability of data and materials}

The datasets used and/or analyzed during the current study are available from the corresponding author on reasonable request.

\section{Authors' contributions}

JWC, SKL, GSB and SJP made substantial contributions to the conception and design of the study; JWC, SKL, MJK, DGK, JYS, ZQZ, HJS and IJJ were involved in data analysis and interpretation; JWC, SKL, HJS, GSB and SJP drafted the manuscript and revised it critically for important intellectual content. All authors read and approved the final manuscript, and agree to be accountable for all aspects of the research in ensuring that the accuracy or integrity of any part of the work are appropriately investigated and resolved.

\section{Ethics approval and consent to participate}

The present study was approved by the Animal Care Committee of Wonkwang University (WKU15-18). All participants provided informed consent.

\section{Patient consent for publication}

Not applicable.

\section{Competing interests}

The authors declare that they have no competing interests.

\section{References}

1. Apte M, Pirola R and Wilson J: The fibrosis of chronic pancreatitis: New insights into the role of pancreatic stellate cells. Antioxid Redox Signal 15: 2711-2722, 2011.

2. Hobbs PM, Johnson WG and Graham DY: Management of pain in chronic pancreatitis with emphasis on exogenous pancreatic enzymes. World J Gastrointest Pharmacol Ther 7: 370-386, 2016.

3. Yang AL, Vadhavkar S, Singh G and Omary MB: Epidemiology of alcohol-related liver and pancreatic disease in the United States. Arch Intern Med 168: 649-656, 2008.

4. Robles-Diaz G, Vargas F, Uscanga L and Fernández-del Castillo C: Chronic pancreatitis in Mexico City. Pancreas 5: 479-483, 1990.

5. Hirota M, Shimosegawa T, Masamune A, Kikuta K, Kume K, Hamada S, Kihara Y, Satoh A, Kimura K, Tsuji I, et al: The sixth nationwide epidemiological survey of chronic pancreatitis in Japan. Pancreatology 12: 79-84, 2012.

6. Miyake H, Harada H, Ochi K, Kunichika K, Tanaka J and Kimura I: Prognosis and prognostic factors in chronic pancreatitis. Dig Dis Sci 34: 449-455, 1989.

7. Ammann RW, Akovbiantz A, Largiader F and Schueler G: Course and outcome of chronic pancreatitis. Longitudinal study of a mixed medical-surgical series of 245 patients. Gastroenterology 86: 820-828, 1984.

8. Lowenfels AB, Maisonneuve P, Cavallini G, Ammann RW, Lankisch PG, Andersen JR, Dimagno EP, Andrén-Sandberg A and Domellöf L: Pancreatitis and the risk of pancreatic cancer. International pancreatitis study group. N Engl J Med 328: 1433-1437, 1993.

9. Haber PS, Keogh GW, Apte MV, Moran CS, Stewart NL, Crawford DH, Pirola RC, McCaughan GW, Ramm GA and Wilson JS: Activation of pancreatic stellate cells in human and experimental pancreatic fibrosis. Am J Pathol 155: 1087-1095, 1999.

10. Aghdassi AA, Mayerle J, Christochowitz S, Weiss FU, Sendler M and Lerch MM: Animal models for investigating chronic pancreatitis. Fibrogenesis Tissue Repair 4: 26, 2011.

11. Turner R: Acute pancreatitis is a chronic disease. Pancreatic Dis Ther 3: 118, 2013.

12. Wehr AY, Furth EE, Sangar V, Blair IA and Yu KH: Analysis of the human pancreatic stellate cell secreted proteome. Pancreas 40: 557-566, 2011.

13. Schneider A, Löhr JM and Singer MV: The M-ANNHEIM classification of chronic pancreatitis: Introduction of a unifying classification system based on a review of previous classifications of the disease. J Gastroenterol 42: 101-119, 2007.

14. Lankisch PG, Löhr-Happe A, Otto J and Creutzfeldt W: Natural course in chronic pancreatitis. Pain, exocrine and endocrine pancreatic insufficiency and prognosis of the disease. Digestion 54: 148-155, 1993.

15. Singh YN: Kava: An overview. J Ethnopharmacol 37: 13-45, 1992.

16. Srinivasan K: Black pepper and its pungent principle-piperine: A review of diverse physiological effects. Crit Rev Food Sci Nutr 47: 735-748, 2007.

17. Bae GS, Kim MS, Jeong J, Lee HY, Park KC, Koo BS, Kim BJ, Kim TH, Lee SH, Hwang SY, et al: Piperine ameliorates the severity of cerulein-induced acute pancreatitis by inhibiting the activation of mitogen activated protein kinases. Biochem Biophys Res Commun 410: 382-390, 2011.

18. Bae GS, Kim MS, Jung WS, Seo SW, Yun SW, Kim SG, Park RK, Kim EC, Song HJ and Park SJ: Inhibition of lipopolysaccharide-induced inflammatory responses by piperine. Eur J Pharmacol 642: 154-162, 2010.

19. Van Laethem JL, Robberecht P, Résibois A and Deviére J: Transforming growth factor beta promotes development of fibrosis after repeated courses of acute pancreatitis in mice. Gastroenterology 110: 576-582, 1996.

20. Livak KJ and Schmittgen TD: Analysis of relative gene expression data using real-time quantitative PCR and the 2(-Delta Delta C(T)) method. Methods 25: 402-408, 2001.

21. Masamune A, Watanabe T, Kikuta K and Shimosegawa T: Roles of pancreatic stellate cells in pancreatic inflammation and fibrosis. Clin Gastroenterol Hepatol 7 (11 Suppl): S48-S54, 2009. 
22. Vinokurova LV, Zhivaeva NS, Tsaregorodtseva TM and Serova TI: A cytokine status in chronic alcoholic and biliary pancreatitis. Ter Arkh 78: 57-60, 2006 (In Russian).

23. Zhang H, Neuhöfer P, Song L, Rabe B, Lesina M, Kurkowski MU, Treiber M, Wartmann T, Regnér S, Thorlacius H, et al: IL-6 trans-signaling promotes pancreatitis-associated lung injury and lethality. J Clin Invest 123: 1019-1031, 2013.

24. Shek FW, Benyon RC, Walker FM, McCrudden PR, Pender SL, Williams EJ, Johnson PA, Johnson CD, Bateman AC, Fine DR and Iredale JP: Expression of transforming growth factor-beta 1 by pancreatic stellate cells and its implications for matrix secretion and turnover in chronic pancreatitis. Am J Pathol 160 $1787-1798,2002$

25. Vogelmann R, Ruf D, Wagner M, Adler G and Menke A: Effects of fibrogenic mediators on the development of pancreatic fibrosis in a TGF-beta1 transgenic mouse model. Am J Physiol Gastrointest Liver Physiol 280: G164-G172, 2001.

26. Gressner AM, Weiskirchen R, Breitkopf K and Dooley S: Roles of TGF-beta in hepatic fibrosis. Front Biosci 7: d793-d807, 2002.

27. Chung H, Ramachandran R, Hollenberg MD and Muruve DA: Proteinase-activated receptor-2 transactivation of epidermal growth factor receptor and transforming growth factor- $\beta$ receptor signaling pathways contributes to renal fibrosis. J Biol Chem 288 37319-37331, 2013.

28. Fu M, Sun ZH and Zuo HC: Neuroprotective effect of piperine on primarily cultured hippocampal neurons. Biol Pharm Bull 33: 598-603, 2010

29. Bang JS, Oh DH, Choi HM, Sur BJ, Lim SJ, Kim JY, Yang HI, Yoo MC, Hahm DH and Kim KS: Anti-inflammatory and antiarthritic effects of piperine in human interleukin 1beta-stimulated fibroblast-like synoviocytes and in rat arthritis models. Arthritis Res Ther 11: R49, 2009.

30. Taqvi SI, Shah AJ and Gilani AH: Blood pressure lowering and vasomodulator effects of piperine. J Cardiovasc Pharmacol 52: 452-458, 2008

31. Mehmood MH and Gilani AH: Pharmacological basis for the medicinal use of black pepper and piperine in gastrointestinal disorders. J Med Food 13: 1086-1096, 2010.

32. Diwan V, Poudyal H and Brown L: Piperine attenuates cardiovascular, liver and metabolic changes in high carbohydrate, high fat-fed rats. Cell Biochem Biophys 67: 297-304, 2013.

33. Durgaprasad S, Pai CG, Vasanthkumar, Alvres JF and Namitha $S$ A pilot study of the antioxidant effect of curcumin in tropical pancreatitis. Indian J Med Res 122: 315-318, 2005.

34. Schneider E, Schmid-Kotsas A, Zhao J, Weidenbach H, Schmid RM, Menke A, Adler G, Waltenberger J, Grünert A and Bachem MG: Identification of mediators stimulating proliferation and matrix synthesis of rat pancreatic stellate cells. Am J Physiol Cell Physiol 281: C532-C543, 2001.
35. Omary MB, Lugea A, Lowe AW and Pandol SJ: The pancreatic stellate cell: A star on the rise in pancreatic diseases. J Clin Invest 117: 50-59, 2007.

36. Apte MV, Pirola RC and Wilson JS: Pancreatic stellate cells: A starring role in normal and diseased pancreas. Front Physiol 3: 344, 2012.

37. Chen B, Li J, Fellows GF, Sun Z and Wang R: Maintaining human fetal pancreatic stellate cell function and proliferation require $\beta 1$ integrin and collagen I matrix interactions. Oncotarget 6 : 14045-14059, 2015.

38. Prud'homme GJ: Pathobiology of transforming growth factor beta in cancer, fibrosis and immunologic disease, and therapeutic considerations. Lab Invest 87: 1077-1091, 2007.

39. Czaja MJ, Weiner FR, Flanders KC, Giambrone MA, Wind R, Biempica $L$ and Zern MA: In vitro and in vivo association of transforming growth factor-beta 1 with hepatic fibrosis. J Cell Biol 108: 2477-2482, 1989.

40. Ma ZG, Yuan YP, Zhang X, Xu SC, Wang SS and Tang QZ Piperine attenuates pathological cardiac fibrosis via PPAR- $\gamma / \mathrm{AKT}$ pathways. EBioMedicine 18: 179-187, 2017

41. Yang L, Shen J, He S, Hu G, Shen J, Wang F, Xu L, Dai W, Xiong $\mathrm{J}$, Ni J, et al: L-cysteine administration attenuates pancreatic fibrosis induced by TNBS in rats by inhibiting the activation of pancreatic stellate cell. PLoS One 7: e31807, 2012.

42. Fabregat I, Moreno-Càceres J, Sánchez A, Dooley S, Dewidar B Giannelli G and Ten Dijke P; IT-LIVER Consortium: TGF- $\beta$ signaling and liver disease. FEBS J 283: 2219-2232, 2016.

43. Wynn TA: Cellular and molecular mechanisms of fibrosis. J Pathol 214: 199-210, 2008.

44. Ohnishi H, Miyata T, Yasuda H, Satoh Y, Hanatsuka K, Kita H, Ohashi A, Tamada K, Makita N, Iiri T, et al: Distinct roles of Smad2-, Smad3-, and ERK-dependent pathways in transforming growth factor-betal regulation of pancreatic stellate cellular functions. J Biol Chem 279: 8873-8878, 2004.

45. Xu L, Zheng N, He Q, Li R, Zhang K and Liang T: Puerarin, isolated from Pueraria lobata (Willd.), protects against hepatotoxicity via specific inhibition of the TGF- $\beta 1 /$ Smad signaling pathway, thereby leading to anti-fibrotic effect. Phytomedicine 20: 1172-1179, 2013

This work is licensed under a Creative Commons Attribution-NonCommercial-NoDerivatives 4.0 International (CC BY-NC-ND 4.0) License. 